\title{
Existence and nonexistence of positive solutions for fractional integral boundary value problem with two disturbance parameters
}

\author{
Xiao Wang, Xiping Liu* and Xuejing Deng
}

"Correspondence:

xipingliu@163.com

College of Science, University of

Shanghai for Science and

Technology, Shanghai, 200093, China

\begin{abstract}
In this paper, we consider a class of fractional differential equations with integral boundary conditions which involve two disturbance parameters. By using the Guo-Krasnoselskii fixed point theorem, new results on the existence and nonexistence of positive solutions for the boundary value problem are obtained. And the impact of the disturbance parameters on the existence of positive solutions is also investigated. Finally, we give some examples to illustrate our main results.

MSC: $34 \mathrm{~B} 15 ; 26 \mathrm{~A} 33$

Keywords: fractional differential equation; Riemann-Liouville fractional derivative; integral boundary problem; positive solution; existence and nonexistence; disturbance parameter
\end{abstract}

\section{Introduction}

The theory of boundary value problems for ordinary differential equations and functional differential equations plays an important role in many research fields of science and engineering; for details, see [1-9] and the references therein. Meanwhile, fractional differential equations have also widely appeared in various fields such as physics, mechanics, electricity, biology, control theory, etc. Therefore, the study of fractional differential equations has gained prominence and has been growing rapidly, see[10-18]. Last but not least, as an important part of fractional differential equations, the integral boundary value problems have also been extensively researched, see [19-23].

In [21], Jia and Liu investigated the existence and nonexistence of positive solutions for the following integral boundary value problem of fractional differential equations with a disturbance parameter in the boundary conditions and the impact of the disturbance parameter on the existence of positive solutions

$$
\left\{\begin{array}{l}
-{ }^{\mathrm{C}} D^{\delta} u(t)=f(t, u(t)), \quad t \in(0,1), \\
m_{1} u(0)-n_{1} u^{\prime}(0)=0, \\
m_{2} u(1)+n_{2} u^{\prime}(1)=\int_{0}^{1} g(s) u(s) \mathrm{d} s+a,
\end{array}\right.
$$

(c) 2015 Wang et al. This article is distributed under the terms of the Creative Commons Attribution 4.0 International License (http://creativecommons.org/licenses/by/4.0/), which permits unrestricted use, distribution, and reproduction in any medium, provided you give appropriate credit to the original author(s) and the source, provide a link to the Creative Commons license, and indicate if changes were made. 
where ${ }^{\mathrm{C}} D^{\delta}$ is the Caputo fractional derivative, $1<\delta \leq 2, f \in C\left([0,1] \times \mathbb{R}^{+}, \mathbb{R}^{+}\right)$and $g \in$ $C\left([0,1], \mathbb{R}^{+}\right)$.

In this paper, we are concerned with the Riemann-Liouville fractional differential equation

$$
D_{0+}^{\alpha} x(t)=f(t, x(t)), \quad t \in(0,1),
$$

with the integral boundary conditions

$$
\left\{\begin{array}{l}
x(0)=x^{\prime}(0)=0, \\
x(1)=\int_{0}^{1} g_{1}(s) x(s) \mathrm{d} s+a, \\
x^{\prime}(1)=\int_{0}^{1} g_{2}(s) x(s) \mathrm{d} s-b,
\end{array}\right.
$$

where $D_{0+}^{\alpha}$ is the standard Riemann-Liouville fractional derivative of order $\alpha, 3<\alpha \leq 4$, $f:[0,1] \times[0,+\infty) \rightarrow[0,+\infty)$ is a continuous function, $g_{1}, g_{2} \in L^{1}[0,1]$, and $a, b \geq 0$. The existence and nonexistence of positive solutions for the integral boundary value problem (1.1)-(1.2) and the impact of the disturbance parameters $a, b$ on the existence of positive solutions is also investigated. Finally, we give two examples to illustrate our results.

\section{Preliminaries}

In this section, we present some useful definitions and related lemmas.

Definition 2.1 (See [12]) Let $\alpha>0$. The fractional integral operator of a function $y$ : $(0,+\infty) \rightarrow \mathbb{R}$ is given by

$$
I^{\alpha} y(t)=\frac{1}{\Gamma(\alpha)} \int_{0}^{t}(t-s)^{\alpha-1} y(s) \mathrm{d} s
$$

provided the integral exists.

Definition 2.2 (See [12]) Let $\alpha>0$. The Riemann-Liouville fractional derivative of a function $y:(0,+\infty) \rightarrow \mathbb{R}$ is given by

$$
D_{0+}^{\alpha} y(t)=D^{z} I_{0+}^{z-\alpha} y(t)=\frac{1}{\Gamma(z-\alpha)}\left(\frac{\mathrm{d}}{\mathrm{d} t}\right)^{z} \int_{0}^{t} \frac{y(s)}{(t-s)^{\alpha-z+1}} \mathrm{~d} s,
$$

where $z \in \mathbb{N}, z-1<\alpha<z$, provided the right-hand side is pointwise defined on $(0, \infty)$.

Lemma 2.1 (See [12]) For $\alpha>0, z \in \mathbb{N}$ and $z-1<\alpha<z$, if $x \in L^{1}[0,1]$ and $I_{0+}^{z-\alpha} x \in A C^{z}[0,1]$, we have the equation

$$
I_{0+}^{\alpha} D_{0+}^{\alpha} x(t)=x(t)+c_{1} t^{\alpha-1}+c_{2} t^{\alpha-2}+c_{3} t^{\alpha-3}+\cdots+c_{z} t^{\alpha-z},
$$

where $c_{i} \in \mathbb{R}, i=1,2,3, \ldots, z$.

Lemma 2.2 The boundary value problem (1.1)-(1.2) is equivalent to the following integral equation:

$$
\begin{aligned}
x(t)= & \int_{0}^{1} G(t, s) f(s, x(s)) \mathrm{d} s+\int_{0}^{1} H(t, s) x(s) \mathrm{d} s \\
& +((\alpha-1) a+b) t^{\alpha-2}-((\alpha-2) a+b) t^{\alpha-1},
\end{aligned}
$$


where

$$
G(t, s)=\frac{1}{\Gamma(\alpha)}\left\{\begin{array}{cc}
(t-s)^{\alpha-1}+(1-s)^{\alpha-2} t^{\alpha-2} & \\
\times((s-t)+(\alpha-2)(1-t) s), & 0 \leq s<t \leq 1, \\
(1-s)^{\alpha-2} t^{\alpha-2}((s-t)+(\alpha-2)(1-t) s), & 0 \leq t<s \leq 1
\end{array}\right.
$$

and

$$
H(t, s)=t^{\alpha-2}(2 t-\alpha t+\alpha-1) g_{1}(s)+t^{\alpha-2}(t-1) g_{2}(s) .
$$

Proof Assume that $x=x(t)$ is a solution of (1.1), it follows from Lemma 2.1 that

$$
x(t)=\frac{1}{\Gamma(\alpha)} \int_{0}^{t}(t-s)^{\alpha-1} f(s, x(s)) \mathrm{d} s+c_{1} t^{\alpha-1}+c_{2} t^{\alpha-2}+c_{3} t^{\alpha-3}+c_{4} t^{\alpha-4},
$$

where $c_{i} \in \mathbb{R}, i=1,2,3,4$.

From the boundary conditions $x(0)=x^{\prime}(0)=0$, we get $c_{3}=c_{4}=0$.

And from the boundary conditions $x(1)=\int_{0}^{1} g_{1}(s) x(s) \mathrm{d} s+a$ and $x^{\prime}(1)=\int_{0}^{1} g_{2}(s) x(s) \mathrm{d} s-b$, we can get

$$
\begin{aligned}
c_{1}= & (2-\alpha) \int_{0}^{1} g_{1}(s) x(s) \mathrm{d} s+\int_{0}^{1} g_{2}(s) x(s) \mathrm{d} s \\
& -\frac{1}{\Gamma(\alpha)} \int_{0}^{1}(1-s)^{\alpha-2}(\alpha s-2 s+1) f(s, x(s)) \mathrm{d} s-b-(\alpha-2) a \\
c_{2}= & (\alpha-1) \int_{0}^{1} g_{1}(s) x(s) \mathrm{d} s-\int_{0}^{1} g_{2}(s) x(s) \mathrm{d} s \\
& +\frac{1}{\Gamma(\alpha-1)} \int_{0}^{1}(1-s)^{\alpha-2} s f(s, x(s)) \mathrm{d} s+b+(\alpha-1) a .
\end{aligned}
$$

Then

$$
\begin{aligned}
x(t)= & \frac{1}{\Gamma(\alpha)} \int_{0}^{t}(t-s)^{\alpha-1} f(s, x(s)) \mathrm{d} s+t^{\alpha-1}(2-\alpha) \int_{0}^{1} g_{1}(s) x(s) \mathrm{d} s+t^{\alpha-1} \int_{0}^{1} g_{2}(s) x(s) \mathrm{d} s \\
& -\frac{t^{\alpha-1}}{\Gamma(\alpha)} \int_{0}^{1}(1-s)^{\alpha-2}(\alpha s-2 s+1) f(s, x(s)) \mathrm{d} s \\
& +t^{\alpha-2}(\alpha-1) \int_{0}^{1} g_{1}(s) x(s) \mathrm{d} s-t^{\alpha-2} \int_{0}^{1} g_{2}(s) x(s) \mathrm{d} s \\
& +\frac{t^{\alpha-2}}{\Gamma(\alpha-1)} \int_{0}^{1}(1-s)^{\alpha-2} s f(s, x(s)) \mathrm{d} s+((\alpha-1) a+b) t^{\alpha-2}-((\alpha-2) a+b) t^{\alpha-1} \\
= & \frac{1}{\Gamma(\alpha)} \int_{0}^{t}\left((t-s)^{\alpha-1}+(1-s)^{\alpha-2} t^{\alpha-2}((s-t)+(\alpha-2)(1-t) s)\right) f(s, x(s)) \mathrm{d} s \\
& +\frac{1}{\Gamma(\alpha)} \int_{t}^{1}(1-s)^{\alpha-2} t^{\alpha-2}((s-t)+(\alpha-2)(1-t) s) f(s, x(s)) \mathrm{d} s \\
& +t^{\alpha-2}(2 t-\alpha t+\alpha-1) \int_{0}^{1} g_{1}(s) x(s) \mathrm{d} s+t^{\alpha-2}(t-1) \int_{0}^{1} g_{2}(s) x(s) \mathrm{d} s \\
& +((\alpha-1) a+b) t^{\alpha-2}-((\alpha-2) a+b) t^{\alpha-1}
\end{aligned}
$$




$$
\begin{aligned}
= & \int_{0}^{1} G(t, s) f(s, x(s)) \mathrm{d} s+\int_{0}^{1} H(t, s) x(s) \mathrm{d} s \\
& +((\alpha-1) a+b) t^{\alpha-2}-((\alpha-2) a+b) t^{\alpha-1} .
\end{aligned}
$$

Hence, $x=x(t)$ is a solution of the integral equation (2.1) if it is the solution of the boundary value problem (1.1)-(1.2), and vice versa.

The proof is completed.

\section{Lemma 2.3 Let $G$ be defined by (2.2), then}

(1) $G(t, s) \in C[0,1] \times[0,1]$, and $G(t, s)>0$ for any $t, s \in(0,1)$,

(2) $(\alpha-2) q(t) k(s) \frac{1}{\Gamma(\alpha)} \leq G(t, s) \leq M_{0} k(s) \frac{1}{\Gamma(\alpha)} \leq M_{0} \frac{1}{\Gamma(\alpha)}$ for any $t, s \in[0,1]$, where

$$
M_{0}=\max \left\{\alpha-1,(\alpha-2)^{2}\right\}, \quad q(t)=t^{\alpha-2}(1-t)^{2}, \quad k(s)=s^{2}(1-s)^{\alpha-2} .
$$

Proof (1) It is easy to show that the result holds.

(2) For $s \leq t$, we could use the mean value theorem of differential calculus and get

$$
\begin{aligned}
G(t, s) & =\frac{1}{\Gamma(\alpha)}\left((t-s)\left((t-s)^{\alpha-2}-(t-t s)^{\alpha-2}\right)+(\alpha-2)(1-t) s(t-t s)^{\alpha-2}\right) \\
& \geq \frac{1}{\Gamma(\alpha)}\left(-(t-s)(\alpha-2)(t-t s)^{\alpha-3} s(1-t)+(\alpha-2)(1-t) s(t-t s)^{\alpha-2}\right) \\
& \geq \frac{1}{\Gamma(\alpha)}(\alpha-2) t^{\alpha-2}(1-s)^{\alpha-2} s^{2}(1-t)^{2}
\end{aligned}
$$

and

$$
\begin{aligned}
G(t, s) & \leq \frac{1}{\Gamma(\alpha)}\left(-(t-s)(\alpha-2)(t-s)^{\alpha-3} s(1-t)+(\alpha-2)(1-t) s(t-t s)^{\alpha-2}\right) \\
& =\frac{1}{\Gamma(\alpha)}(\alpha-2) s(1-t)\left((t-t s)^{\alpha-2}-(t-s)^{\alpha-2}\right) \\
& \leq \frac{1}{\Gamma(\alpha)}(\alpha-2)^{2} s^{2}(1-t)^{2} t^{\alpha-3}(1-s)^{\alpha-3} \\
& \leq \frac{1}{\Gamma(\alpha)}(\alpha-2)^{2} s^{2}(1-s)^{\alpha-2} \\
& \leq \frac{M_{0}}{\Gamma(\alpha)}
\end{aligned}
$$

For $s \geq t$, we have that

$$
\begin{aligned}
& G(t, s) \geq \frac{1}{\Gamma(\alpha)}(\alpha-2) t^{\alpha-2}(1-s)^{\alpha-2} s(1-t) \geq \frac{1}{\Gamma(\alpha)}(\alpha-2) t^{\alpha-2}(1-s)^{\alpha-2} s^{2}(1-t)^{2}, \\
& G(t, s) \leq \frac{1}{\Gamma(\alpha)} s^{\alpha-2}(1-s)^{\alpha-2}(s+(\alpha-2) s)=\frac{1}{\Gamma(\alpha)} s^{\alpha-1}(1-s)^{\alpha-2}(\alpha-1) \leq \frac{M_{0}}{\Gamma(\alpha)} .
\end{aligned}
$$

The proof is completed.

Now we make the following assumption.

(B0) $g_{1}, g_{2} \in L^{1}[0,1]$ such that $0 \leq \inf _{t, s \in[0,1]} H(t, s)<\sup _{t, s \in[0,1]} H(t, s):=M<1$. 
Let the Banach space $C[0,1]$ be endowed with the norm $\|x\|:=\max _{t \in[0,1]}|x(t)|$, and let

$$
P=\{x \in C[0,1]: x(t) \geq 0, t \in[0,1]\},
$$

then $P$ is a cone in $C[0,1]$.

We define an operator $A: P \rightarrow C[0,1]$ by

$$
(A x)(t)=\int_{0}^{1} H(t, s) x(s) \mathrm{d} s .
$$

Lemma 2.4 Assume (B0) holds, then the operator A satisfies the following properties:

(1) $A$ is a bounded linear operator;

(2) $A(P) \subset P$;

(3) the operator $A$ is reversible;

(4) $\left\|(I-A)^{-1}\right\| \leq \frac{1}{1-M}$.

Proof By (B0), it is obvious that (1), (2) hold.

(3) Since $M<1$, we get that $\|A x\| \leq M\|x\|<\|x\|$, then $\|A\| \leq M<1$, so that $I-A$ is reversible.

(4) Let $y(t)=x(t)-A x(t)$, that is, $x(t)=y(t)+A x(t), x(t)=(I-A)^{-1} y(t)$, and $y \in C[0,1]$ for $t \in[0,1]$. From the definition of operator $A$, we have that

$$
x(t)=y(t)+\int_{0}^{1} H(t, s) x(s) \mathrm{d} s .
$$

Let

$$
x_{0}(t)=x(t), \quad x_{m}(t)=y(t)+\int_{0}^{1} H(t, s) x_{m-1}(s) \mathrm{d} s, \quad m=1,2, \ldots
$$

We apply the method of iteration to solve the above equation.

According to this method, we can get that

$$
x(t)=(I-A)^{-1} y(t)=y(t)+\int_{0}^{1} R(t, s) y(s) \mathrm{d} s,
$$

where

$$
\begin{aligned}
& R(t, s)=\sum_{j=1}^{\infty} H_{j}(t, s), \quad H_{1}(t, s)=H(t, s) \quad \text { and } \\
& H_{j}(t, s)=\int_{0}^{1} H(t, \tau) H_{j-1}(\tau, s) \mathrm{d} \tau, \quad j=2,3, \ldots
\end{aligned}
$$

Because of $0 \leq H(t, s)<M<1$, we have that

$$
0 \leq R(t, s)=\sum_{j=1}^{\infty} H_{j}(t, s)<M+M^{2}+\cdots+M^{n}+\cdots=\frac{M}{1-M} .
$$

Since $(I-A)^{-1} y(t)=x(t)$, we get

$$
\left|(I-A)^{-1} y(t)\right| \leq|y(t)|+\frac{M}{1-M}\left|\int_{0}^{1} y(s) \mathrm{d} s\right| \leq\|y\|+\frac{M}{1-M}\|y\|=\frac{\|y\|}{1-M}
$$


and

$$
\left\|(I-A)^{-1} y\right\|=\max _{t \in[0,1]}\left|(I-A)^{-1} y(t)\right| \leq \frac{\|y\|}{1-M} .
$$

So

$$
\left\|(I-A)^{-1}\right\| \leq \frac{1}{1-M}
$$

The proof is completed.

We define another operator $T: P \rightarrow C[0,1]$,

$$
(T x)(t)=\int_{0}^{1} G(t, s) f(s, x(s)) \mathrm{d} s+((\alpha-1) a+b) t^{\alpha-2}-((\alpha-2) a+b) t^{\alpha-1}
$$

It can be easily shown that $T: P \rightarrow P . x(t)$ is a solution of the boundary value problem (1.1)-(1.2) if and only if it satisfies

$$
x(t)=(T x)(t)+(A x)(t) .
$$

Hence,

$$
x(t)=(I-A)^{-1}(T x)(t)=(T x)(t)+\int_{0}^{1} R(t, s)(T x)(s) \mathrm{d} s
$$

and

$$
\begin{aligned}
(I-A)^{-1}(T x)(t)= & \int_{0}^{1} G(t, s) f(s, x(s)) \mathrm{d} s+\int_{0}^{1} R(t, s) \int_{0}^{1} G(s, \tau) f(\tau, x(\tau)) \mathrm{d} \tau \mathrm{d} s \\
& +\int_{0}^{1} R(t, s)\left(((\alpha-1) a+b) s^{\alpha-2}-((\alpha-2) a+b) s^{\alpha-1}\right) \mathrm{d} s \\
& +((\alpha-1) a+b) t^{\alpha-2}-((\alpha-2) a+b) t^{\alpha-1} .
\end{aligned}
$$

Let

$$
P_{0}=\left\{x \in P: x(t) \geq \frac{q(t)(1-M)}{M_{0}}\|x\|, t \in[0,1]\right\} .
$$

Lemma 2.5 Assume that condition (B0) holds, then the operator $(I-A)^{-1} T: P \rightarrow P_{0}$ is completely continuous.

Proof From the continuity and the non-negativeness of functions $G, R$ and $f$, we have that if $x \in P$, then $(I-A)^{-1}(T x)(t) \geq 0$ and $(I-A)^{-1}(T x) \in P$.

It follows from (2.4) and Lemma 2.3, for $x \in P$ and $t \in[0,1]$,

$$
\begin{aligned}
& \left|(I-A)^{-1}(T x)(t)\right| \\
& \quad \leq\left\|(I-A)^{-1}\right\| \cdot|T x(t)|
\end{aligned}
$$




$$
\begin{aligned}
& \leq \frac{1}{1-M}\left|\int_{0}^{1} \frac{M_{0}}{\Gamma(\alpha)} k(s) f(s, x(s)) \mathrm{d} s+((\alpha-1) a+b) t^{\alpha-2}-((\alpha-2) a+b) t^{\alpha-1}\right| \\
& \leq \frac{1}{1-M}\left(\int_{0}^{1} \frac{M_{0}}{\Gamma(\alpha)} k(s) f(s, x(s)) \mathrm{d} s+t^{\alpha-2}((\alpha-2) a(1-t)+b(1-t)+a)\right) .
\end{aligned}
$$

Hence,

$$
\left\|(I-A)^{-1}(T x)\right\| \leq \frac{M_{0}}{1-M}\left(\frac{1}{\Gamma(\alpha)} \int_{0}^{1} k(s) f(s, x(s)) \mathrm{d} s+(\alpha-1) a+b\right) .
$$

By (2.4), (2.5) and (2.6), we have

$$
\begin{aligned}
(I-A)^{-1}(T x)(t) \geq & (T x)(t) \\
\geq & \frac{(\alpha-2) q(t)}{\Gamma(\alpha)} \int_{0}^{1} k(s) f(s, x(s)) \mathrm{d} s \\
& +((\alpha-1) a+b) t^{\alpha-2}-((\alpha-1) a+b) t^{\alpha-1} \\
= & \frac{(\alpha-2) q(t)}{\Gamma(\alpha)} \int_{0}^{1} k(s) f(s, x(s)) \mathrm{d} s+((\alpha-1) a+b) t^{\alpha-2}(1-t) \\
\geq & \frac{q(t)}{\Gamma(\alpha)} \int_{0}^{1} k(s) f(s, x(s)) \mathrm{d} s+((\alpha-1) a+b) t^{\alpha-2}(1-t)^{2} \\
= & q(t)\left(\frac{1}{\Gamma(\alpha)} \int_{0}^{1} k(s) f(s, x(s)) \mathrm{d} s+(\alpha-1) a+b\right) .
\end{aligned}
$$

It follows from (2.7) that

$$
(I-A)^{-1}(T x)(t) \geq \frac{q(t)(1-M)}{M_{0}}\left\|(I-A)^{-1} T x\right\| .
$$

Hence $(I-A)^{-1} T(P) \subset P_{0}$.

Let $\left\{x_{n}\right\} \subset P, x \in P$, and $\left\|x_{n}-x\right\| \rightarrow 0$ as $n \rightarrow+\infty$, there exists a constant $r>0$ such that $\left\|x_{n}\right\| \leq r$ and $\|x\| \leq r$. We have

$$
\lim _{n \rightarrow \infty} f\left(s, x_{n}(s)\right)=f(s, x(s)) \quad \text { for a.e. } s \in[0,1]
$$

By the Lebesgue dominated convergence theorem, we get

$$
\lim _{n \rightarrow \infty}(I-A)^{-1}\left(T x_{n}\right)(t)=(I-A)^{-1}(T x)(t)
$$

So

$$
\lim _{n \rightarrow \infty}\left\|(I-A)^{-1} T x_{n}-(I-A)^{-1} T x\right\|=0 .
$$

Then the operator $(I-A)^{-1} T$ is continuous.

Let $\Omega \subset P$ be bounded. Then there exists a positive constant $l>0$ such that $\|x\| \leq l$ for all $x \in \Omega$. Let $N=\max _{0 \leq t \leq 1,0 \leq x \leq l}|f(t, x)|+1$. By (2.7), for all $x \in \Omega$, we have

$$
\left\|(I-A)^{-1}(T x)(t)\right\| \leq \frac{M_{0}}{(1-M)}\left(\frac{N}{\Gamma(\alpha)}+(\alpha-1) a+b\right)
$$

which means $(I-A)^{-1} T(\Omega)$ is bounded in $P$. 
In addition, for any given $x \in \Omega$, because $G(t, s)$ is continuous for $(t, s) \in[0,1] \times[0,1]$, then it must be uniformly continuous. So, for any $\varepsilon>0$, there exists a constant $\delta_{0}>0$ such that for all $s \in[0,1]$, as $\left|t_{1}-t_{2}\right|<\delta_{0}$, we have that

$$
\begin{aligned}
& \left|G\left(t_{1}, s\right)-G\left(t_{2}, s\right)\right|<\varepsilon, \\
& \left|t_{1}^{\alpha-2}-t_{2}^{\alpha-2}\right|<\varepsilon, \\
& \left|t_{1}^{\alpha-1}-t_{2}^{\alpha-1}\right|<\varepsilon .
\end{aligned}
$$

For each $x \in \Omega$,

$$
\begin{aligned}
&\left|(I-A)^{-1}(T x)\left(t_{1}\right)-(I-A)^{-1}(T x)\left(t_{2}\right)\right| \\
& \leq\left\|(I-A)^{-1}\right\| \cdot\left|(T x)\left(t_{1}\right)-(T x)\left(t_{2}\right)\right| \\
& \leq \frac{1}{1-M} \int_{0}^{1}\left|G\left(t_{1}, s\right)-G\left(t_{2}, s\right)\right||f(s, x(s))| \mathrm{d} s \\
& \quad+\frac{(\alpha-1) a+b}{1-M}\left|t_{1}^{\alpha-2}-t_{2}^{\alpha-2}\right|+\frac{(\alpha-2) a+b}{1-M}\left|t_{1}^{\alpha-1}-t_{2}^{\alpha-1}\right| \\
&<\frac{N+(2 \alpha-3) a+2 b}{1-M} \varepsilon .
\end{aligned}
$$

We have

$$
\left\|(I-A)^{-1}(T x)\left(t_{1}\right)-(I-A)^{-1}(T x)\left(t_{2}\right)\right\|<\frac{N+(2 \alpha-3) a+2 b}{1-M} \varepsilon,
$$

and $(I-A)^{-1} T(\Omega)$ is equicontinuous in $P_{0}$.

Now, according to the Arzela-Ascoli theorem, we conclude that $(I-A)^{-1} T(\Omega)$ is relatively compact.

Therefore, $(I-A)^{-1} T: P \rightarrow P_{0}$ is a completely continuous operator.

The proof is completed.

By Lemma 2.2, we can easily deduce that the following lemma holds.

Lemma 2.6 Assume $x \in C[0,1], D_{0^{+}}^{\alpha} x \in L^{1}[0,1]$. Then the boundary value problem (1.1)(1.2) has a positive solution if and only if the operator $(I-A)^{-1} T$ has a fixed point in $P$. Furthermore, if $x$ is a positive solution of the fractional boundary value problem (1.1)-(1.2), then $x \in P_{0}$.

To prove the existence of positive solution for the boundary value problem (1.1)-(1.2), we state the following Guo-Krasnoselskii fixed point theorem, see [24].

Lemma 2.7 Let $E$ be a Banach space and $P \subset E$ be a cone. Assume that $\Omega_{1}, \Omega_{2}$ are bounded open subsets of $E$ with $\theta \in \Omega_{1} \subset \bar{\Omega}_{1} \subset \Omega_{2}$, and let $T: P \cap\left(\bar{\Omega}_{2} \backslash \Omega_{1}\right) \rightarrow P$ be a completely continuous operator such that either

(1) $\|T(x)\| \leq\|x\|, \forall x \in P \cap \partial \Omega_{1}$; and $\|T(x)\| \geq\|x\|, \forall x \in P \cap \partial \Omega_{2}$, or

(2) $\|T(x)\| \geq\|x\|, \forall x \in P \cap \partial \Omega_{1}$; and $\|T(x)\| \leq\|x\|, \forall x \in P \cap \partial \Omega_{2}$.

Then the operator $T$ has at least one fixed point in $P \cap\left(\bar{\Omega}_{2} \backslash \Omega_{1}\right)$. 


\section{Existence and nonexistence of positive solutions}

Denote

$$
\begin{aligned}
& f_{0}=\liminf _{x \rightarrow 0^{+}} \inf _{t \in\left[\frac{1}{4}, \frac{3}{4}\right]} \frac{f(t, x)}{x}, \quad f^{0}=\limsup _{x \rightarrow 0^{+}} \sup _{t \in[0,1]} \frac{f(t, x)}{x}, \\
& f_{\infty}=\liminf _{x \rightarrow \infty} \inf _{t \in\left[\frac{1}{4}, \frac{3}{4}\right]} \frac{f(t, x)}{x}, \quad f^{\infty}=\limsup _{x \rightarrow \infty} \sup _{t \in[0,1]} \frac{f(t, x)}{x}, \\
& \sigma=\min _{t \in\left[\frac{1}{4}, \frac{3}{4}\right]} \frac{q(t)(1-M)}{M_{0}}=\frac{(1-M) 3^{\alpha-2}}{M_{0} 4^{\alpha}}, \quad \rho_{1}=\frac{(1-M) \Gamma(\alpha)}{M_{0}+\alpha \Gamma(\alpha)}, \quad \rho_{2}=\frac{7,680 \Gamma(\alpha) 2^{\alpha}}{203 \sigma(\alpha-2)} .
\end{aligned}
$$

Theorem 3.1 Suppose (B0) holds, $f^{0}<\rho_{1}$ and $f_{\infty}>\rho_{2}$. Then there exist small enough $a_{0}$ and $b_{0}$ such that the boundary value problem (1.1)-(1.2) has at least one positive solution for $0 \leq a \leq a_{0}$ and $0 \leq b \leq b_{0}$.

Proof Since $f^{0}<\rho_{1}$, there exists a constant $r_{1}>0$ such that

$$
f(t, x)<\rho_{1} x \leq \rho_{1} r_{1}
$$

for all $t \in[0,1]$ and $x \in\left[0, r_{1}\right]$.

Let $\Omega_{1}=\left\{x \in P_{0}:\|x\|<r_{1}\right\}, 0 \leq a \leq a_{0}, 0 \leq b \leq b_{0}$ and $\max \left\{a_{0}, b_{0}\right\} \leq \rho_{1} r_{1}$.

By Lemma 2.3, for $x \in \partial \Omega_{1}$, we have $\|x\|=r_{1}$ and

$$
\begin{aligned}
0 & \leq(I-A)^{-1}(T x)(t) \leq\left\|(I-A)^{-1}\right\| \cdot|(T x)(t)| \leq \frac{1}{1-M}|(T x)(t)| \\
& \leq \frac{1}{1-M}\left(\frac{M_{0}}{\Gamma(\alpha)} \int_{0}^{1} f(s, x(s)) \mathrm{d} s+(\alpha-1) a+b\right) \\
& \leq \frac{1}{1-M}\left(\frac{M_{0}}{\Gamma(\alpha)} \int_{0}^{1} f(s, x(s)) \mathrm{d} s+(\alpha-1) a_{0}+b_{0}\right) \\
& \leq \frac{M_{0}}{(1-M) \Gamma(\alpha)} \rho_{1}\|x\|+\frac{1}{1-M}\left((\alpha-1) \rho_{1}\|x\|+\rho_{1}\|x\|\right) \\
& =\frac{M_{0}+\alpha \Gamma(\alpha)}{(1-M) \Gamma(\alpha)} \rho_{1}\|x\|=\|x\| .
\end{aligned}
$$

So we get $\left\|(I-A)^{-1} T x\right\| \leq\|x\|, x \in \partial \Omega_{1}$.

Since $f_{\infty}>\rho_{2}$, there exists a constant $R>0$ such that

$$
f(t, x)>\rho_{2} x
$$

for all $t \in\left[\frac{1}{4}, \frac{3}{4}\right]$ and $x \in[R,+\infty)$.

Let $r_{2}>\max \left\{r_{1}, \frac{R}{\sigma}\right\}$ and $\Omega_{2}=\left\{x \in P_{0}:\|x\|<r_{2}\right\}$.

For all $x \in \partial \Omega_{2}$, we have that $\|x\|=r_{2}$ and $x(t) \geq \frac{q(t)(1-M)}{M_{0}}\|x\| \geq \sigma\|x\|=\sigma r_{2}>R$ for $t \in$ $\left[\frac{1}{4}, \frac{3}{4}\right]$. Then

$$
\begin{aligned}
(I-A)^{-1}(T x)\left(\frac{1}{2}\right) & \geq(T x)\left(\frac{1}{2}\right) \geq \int_{0}^{1} G\left(\frac{1}{2}, s\right) f(s, x(s)) \mathrm{d} s \\
& \geq \frac{(\alpha-2) q\left(\frac{1}{2}\right)}{\Gamma(\alpha)} \int_{0}^{1} k(s) f(s, x(s)) \mathrm{d} s
\end{aligned}
$$




$$
\begin{aligned}
& \geq \frac{(\alpha-2) q\left(\frac{1}{2}\right)}{\Gamma(\alpha)} \int_{\frac{1}{4}}^{\frac{3}{4}} k(s) f(s, x(s)) \mathrm{d} s \\
& \geq \frac{(\alpha-2) \sigma \rho_{2}}{2^{\alpha} \Gamma(\alpha)} \int_{\frac{1}{4}}^{\frac{3}{4}} k(s) \mathrm{d} s\|x\| \\
& \geq \frac{(\alpha-2) \sigma \rho_{2}}{2^{\alpha} \Gamma(\alpha)} \int_{\frac{1}{4}}^{\frac{3}{4}}(1-s)^{2} s^{2} \mathrm{~d} s\|x\| \\
& =\frac{203 \sigma(\alpha-2)}{7,680 \Gamma(\alpha) 2^{\alpha}} \rho_{2}\|x\| .
\end{aligned}
$$

So $\left\|(I-A)^{-1} T x\right\| \geq\|x\|, x \in \partial \Omega_{2}$.

By Lemma 2.7, we conclude that the operator $(I-A)^{-1} T$ has at least one fixed point in $P_{0} \cap\left(\bar{\Omega}_{2} \backslash \Omega_{1}\right)$, which implies that the boundary value problem (1.1)-(1.2) has a positive solution.

The proof is completed.

Theorem 3.2 Assume (B0) holds, $f^{\infty}<\rho_{1}$ and $f_{0}>\rho_{2}$. Then there exist small enough $a_{0}$ and $b_{0}$ such that the boundary value problem (1.1)-(1.2) has at least one positive solution for $0 \leq a \leq a_{0}$ and $0 \leq b \leq b_{0}$.

Proof Since $f^{\infty}<\rho_{1}$, for $\varepsilon=\frac{\rho_{1}-f^{\infty}}{2}>0$, there exists a constant $R_{1}>0$ such that $f(t, x)<$ $\left(\rho_{1}-\varepsilon\right) x$ for $t \in[0,1]$ and $x \in\left[R_{1},+\infty\right)$.

Let $L=\max _{(t, x) \in[0,1] \times\left[0, R_{1}\right]} f(t, x)$, so

$$
f(t . x) \leq L+\left(\rho_{1}-\varepsilon\right) x \quad \text { for } t \in[0,1] \text { and } x \in[0,+\infty)
$$

Let $r_{3}>\max \left\{R_{1}, \frac{L}{\varepsilon}\right\}, \Omega_{3}=\left\{x \in P_{0}:\|x\|<r_{3}\right\}, 0 \leq a \leq a_{0}, 0 \leq b \leq b_{0}$ and $\max \left\{a_{0}, b_{0}\right\} \leq$ $\rho_{1} r_{3}$.

For all $x \in \partial \Omega_{3}$, we have $\|x\|=r_{3}$ and

$$
\begin{aligned}
\left|(I-A)^{-1}(T x)(t)\right| & \leq\left\|(I-A)^{-1}\right\| \cdot|(T x)(t)| \leq \frac{1}{1-M}|(T x)(t)| \\
& \leq \frac{1}{1-M}\left(\int_{0}^{1} \frac{M_{0}}{\Gamma(\alpha)} f(s, x(s)) \mathrm{d} s+(\alpha-1) a+b\right) \\
& \leq \frac{1}{1-M}\left(\int_{0}^{1} \frac{M_{0}}{\Gamma(\alpha)} f(s, x(s)) \mathrm{d} s+(\alpha-1) a_{0}+b_{0}\right) \\
& \leq \frac{M_{0}}{(1-M) \Gamma(\alpha)} \int_{0}^{1}\left(L+\left(\rho_{1}-\varepsilon\right)\|x\|\right) \mathrm{d} s+\frac{\alpha}{1-M} \rho_{1}\|x\| \\
& \leq \frac{M_{0}+\alpha \Gamma(\alpha)}{(1-M) \Gamma(\alpha)} \rho_{1}\|x\|=\|x\| .
\end{aligned}
$$

So $\left\|(I-A)^{-1} T x\right\| \leq\|x\|, x \in \partial \Omega_{3}$.

Since $f_{0}>\rho_{2}$, there exists a constant $0<r_{4}<R_{1}$ such that $f(t, x)>\rho_{2} x$ for $t \in\left[\frac{1}{4}, \frac{3}{4}\right]$ and $x \in\left[0, r_{4}\right]$.

Let $\Omega_{4}=\left\{x \in P_{0}:\|x\|<r_{4}\right\}$. Similar to the proof of Theorem 3.1, we show $\left\|(I-A)^{-1} T x\right\| \geq$ $\|x\|, x \in \partial \Omega_{4}$. 
By Lemma 2.7, we conclude that the operator $(I-A)^{-1} T$ has at least one fixed point in $P_{0} \cap\left(\bar{\Omega}_{2} \backslash \Omega_{1}\right)$, which implies that the boundary value problem (1.1)-(1.2) has at least one positive solution.

The proof is completed.

Theorem 3.3 Suppose (B0) holds, $f_{\infty}>\rho_{2}$. Then there exist large enough positive constants $a_{1}$ and $b_{1}$ such that the boundary value problem (1.1)-(1.2) has no positive solution for $a>a_{1}$ and $b>b_{1}$.

Proof Assume that for any large enough $a>0$ and $b>0$, the boundary value problem (1.1)-(1.2) has a positive solution $x(t)$.

Since $f_{\infty}>\rho_{2}$, there exists a large enough constant $R_{0}>0$ such that

$$
f(t, x)>\rho_{2} x \quad \text { for } x \in\left[\sigma R_{0},+\infty\right) \text { and } t \in\left[\frac{1}{4}, \frac{3}{4}\right] .
$$

Let $\min \left\{a_{1}, b_{1}\right\}>2^{\alpha-1} R_{0}, a>a_{1}$ and $b>b_{1}$. So $\alpha a+b>\alpha a_{1}+b_{1}>(\alpha+1) 2^{\alpha-1} R_{0}>2^{\alpha-1} R_{0}$. By (2.1), Lemma 2.3 and (B0), we have

$$
x(t) \geq((\alpha-1) a+b) t^{\alpha-2}-((\alpha-2) a+b) t^{\alpha-1} .
$$

Hence,

$$
\begin{aligned}
x\left(\frac{1}{2}\right) & \geq((\alpha-1) a+b)\left(\frac{1}{2}\right)^{\alpha-2}-((\alpha-2) a+b)\left(\frac{1}{2}\right)^{\alpha-1} \\
& =\left(\frac{1}{2}\right)^{\alpha-1}(\alpha a+b)>\left(\frac{1}{2}\right)^{\alpha-1}\left(\alpha a_{1}+b_{1}\right)>R_{0},
\end{aligned}
$$

and we get $\|x\|>R_{0}$.

On the other hand, in view of Lemma 2.6, $x \in P_{0}$. Then $x(t) \geq \sigma\|x\|>\sigma R_{0}$ for $t \in\left[\frac{1}{4}, \frac{3}{4}\right]$. Therefore,

$$
\begin{aligned}
x\left(\frac{1}{2}\right) & =(I-A)^{-1}(T x)\left(\frac{1}{2}\right) \geq(T x)\left(\frac{1}{2}\right) \\
& =\int_{0}^{1} G\left(\frac{1}{2}, s\right) f(s, x(s)) \mathrm{d} s+((\alpha-1) a+b)\left(\frac{1}{2}\right)^{\alpha-2}-((\alpha-2) a+b)\left(\frac{1}{2}\right)^{\alpha-1} \\
& \geq \frac{(\alpha-2) \sigma \rho_{2}}{2^{\alpha} \Gamma(\alpha)} \int_{\frac{1}{4}}^{\frac{3}{4}} k(s) \mathrm{d} s\|x\|+\left(\frac{1}{2}\right)^{\alpha-1}(2((\alpha-1) a+b)-((\alpha-2) a+b)) \\
& \geq \frac{203 \sigma(\alpha-2)}{7,680 \Gamma(\alpha) 2^{\alpha}} \rho_{2}\|x\|+\left(\frac{1}{2}\right)^{\alpha-1}(a(2 \alpha-2-\alpha+2)+b) \\
& \geq\|x\|+\left(\frac{1}{2}\right)^{\alpha-1}(\alpha a+b) \\
& >\|x\|+R_{0} .
\end{aligned}
$$

So $\|x\|>\|x\|+R_{0}$, which is a contradiction. Thus, there exist large enough positive constants $a_{1}$ and $b_{1}$ such that the boundary value problem (1.1)-(1.2) has no positive solution for $a>a_{1}$ and $b>b_{1}$. 


\section{Examples}

To illustrate our main results, we present the following examples.

Example 4.1 We consider the boundary value problem

$$
\left\{\begin{array}{l}
D_{0+}^{\frac{10}{3}} x(t)=x^{\frac{3}{2}}+x^{2} \sin t, \\
x(0)=x^{\prime}(0)=0, \\
x(1)=\frac{1}{3} \int_{0}^{1} x(s) \mathrm{d} s+a, \\
x^{\prime}(1)=\frac{2}{3} \int_{0}^{1} x(s) \mathrm{d} s-b,
\end{array}\right.
$$

and we can establish the following results:

(1) The boundary value problem (4.1) has at least one positive solution if parameters $a \in[0,0.001)$ and $b \in[0,0.001)$.

(2) The boundary value problem (4.1) has no positive solution if parameters $a \in\left(2.15 \times 10^{10},+\infty\right)$ and $b \in\left(2.15 \times 10^{10},+\infty\right)$.

Proof The boundary value problem (4.1) can be regarded as the boundary value problem (1.1)-(1.2), where $\alpha=\frac{10}{3}, g_{1}(s)=\frac{1}{3}, g_{2}(s)=\frac{2}{3}, f(t, x)=x^{\frac{3}{2}}+x^{2} \sin t$.

Then $M=\frac{1}{3}, M_{0}=\frac{7}{3}, \sigma=\min _{t \in\left[\frac{1}{4}, \frac{3}{4}\right]} \frac{q(t)(1-M)}{M_{0}}=\frac{(1-M) 3^{\alpha-2}}{M_{0} 4^{\alpha}}=0.0122, \rho_{1}=\frac{(1-M) \Gamma(\alpha)}{M_{0}+\alpha \Gamma(\alpha)}=0.16$, $\rho_{2}=\frac{7,680 \Gamma(\alpha) 2^{\alpha}}{203 \sigma(\alpha-2)}=65,296.4$ and

$$
f_{\infty}=\infty>\rho_{2}, \quad f^{0}=0<\rho_{1} .
$$

(1) Let $r_{1}=0.0064$, we choose $\max \left\{a_{0}, b_{0}\right\}<\rho_{1} r_{1}=0.001$. When $x \in(0,0.0064], t \in$ $[0,1]$, we have $f(t, x) \leq \rho_{1} r_{1}$. Then, by Theorem 3.1, when $a \in[0,0.001)$ and $b \in[0,0.001)$, the boundary value problem (4.1) has a positive solution.

(2) Let $R_{0}=4.26 \times 10^{9}$, when $x \in\left[5.2 \times 10^{7},+\infty\right)$ and $t \in\left[\frac{1}{4}, \frac{3}{4}\right]$, so we choose $\min \left\{a_{1}, b_{1}\right\}>2^{\alpha-1} R_{0}=2.15 \times 10^{10}$. By Theorem 3.3, for $a \in\left(2.15 \times 10^{10},+\infty\right)$ and $b \in$ $\left(2.15 \times 10^{10},+\infty\right)$, the boundary value problem (4.1) has no positive solution.

Example 4.2 We consider the boundary value problem

$$
\left\{\begin{array}{l}
D_{0+}^{\frac{13}{4}} x(t)=\frac{(t \sqrt{x}+1) x^{\frac{1}{3}}}{2+\sqrt{x}}, \\
x(0)=x^{\prime}(0)=0, \\
x(1)=\frac{1}{2} \int_{0}^{1} x(s) \mathrm{d} s+a, \\
x^{\prime}(1)=\frac{3}{4} \int_{0}^{1} x(s) \mathrm{d} s-b .
\end{array}\right.
$$

The boundary value problem (4.2) has a positive solution for parameters $a \in[0,8.16)$ and $b \in[0,8.16)$.

Proof Where $\alpha=\frac{13}{4}, g_{1}(s)=\frac{1}{2}, g_{2}(s)=\frac{3}{4}, f(t, x)=\frac{(t \sqrt{x}+1) \frac{1}{3}}{2+\sqrt{x}}$, we have that $M_{0}=\frac{9}{4}, M=\frac{1}{2}, \sigma=$ $\min _{t \in\left[\frac{1}{4}, \frac{3}{4}\right]} \frac{q(t)(1-M)}{M_{0}}=\frac{(1-M) 3^{\alpha-2}}{M_{0} 4^{\alpha}}=0.0097, \rho_{1}=\frac{(1-M) \Gamma(\alpha)}{M_{0}+\alpha \Gamma(\alpha)}=0.12$ and $\rho_{2}=\frac{7,680 \Gamma(\alpha) 2^{\alpha}}{203 \sigma(\alpha-2)}=75,722$.

We set $r_{3}=68.04$, when $x \in(68.04,+\infty), t \in[0,1]$, and we choose $\max \left\{a_{0}, b_{0}\right\}<\rho_{1} r_{3}=$ 8.16 for $a \in[0,8.16)$ and $b \in[0,8.16)$. So we have

$$
f_{0}=\infty>\rho_{2}, \quad f^{\infty}=0<\rho_{1} .
$$

By Theorem 3.2, the boundary value problem (4.2) has a positive solution. 


\section{Competing interests}

The authors declare that no competing interests exist.

\section{Authors' contributions}

The authors contributed equally to this paper. All authors read and approved the final manuscript.

\section{Acknowledgements}

This work is supported by the National Natural Science Foundation of China (No. 11171220) and the Hujiang Foundation of China (B14005).

\section{Received: 4 August 2015 Accepted: 30 September 2015 Published online: 12 October 2015}

\section{References}

1. Ge, W: The Boundary Value Problems for Nonlinear Ordinary Differential Equations. Science Press, Beijing (2007) (in Chinese)

2. Lu, S, Ge, W: On the existence of periodic solutions for neutral functional differential equation. Nonlinear Anal. 54, 1285-1306 (2003)

3. Wang, P, Gao, W: Quasilinearization of an initial value problem for a set valued integro-differential equation. Comput. Math. Appl. 61, 2111-2115 (2011)

4. He, Z, Li, L: Multiple positive solutions for the one-dimensional $p$-Laplacian dynamic equations on time scales. Math. Comput. Model. 45, 68-79 (2007)

5. Du, Z, Lv, Y: Permanence and almost periodic solution of a Lotka-Volterra model with mutual interference and time delays. Appl. Math. Model. 37, 1054-1068 (2013)

6. Wang, P, Liu, X: Nonlinear variation of parameters formula for impulsive differential equations with initial time difference and application. Abstr. Appl. Anal. 2014, Article ID 725832 (2014)

7. Lian, H, Zhao, J, Agarwal, RP: Upper and lower solution method for $n$ th-order BVPs on an infinite interval. Bound. Value Probl. 2014, Article ID 100 (2014)

8. Zhang, X, Feng, M: Positive solutions of singular beam equations with the bending term. Bound. Value Probl. 2015, Article ID 84 (2015). doi:10.1186/s13661-015-0348-y

9. Zhao, J, Chu, B, Lian, H: Existence of solutions of multi-point boundary value problems on time scales at resonance. Adv. Differ. Equ. 2013, Article ID 351 (2013)

10. Zhou, Y: Basic Theory of Fractional Differential Equations. World Scientific, Singapore (2014)

11. Podlubny, l: Fractional Differential Equations. Academic Press, New York (1999)

12. Kilbas, AA, Srivastava, HM, Trujillo, JJ: Theory and Applications of Fractional Differential Equations. Elsevier, Amsterdam (2006)

13. Bai, Z: On positive solutions of a nonlocal fractional boundary value problem. Nonlinear Anal. 72, 916-924 (2010)

14. Liu, X, Jia, M, Ge, W: Multiple solutions of a $p$-Laplacian model involving a fractional derivative. Adv. Differ. Equ. 2013 Article ID 126 (2013)

15. $\mathrm{Xu}, \mathrm{X}$, Jiang, $\mathrm{D}$, Yuan, $\mathrm{C}$ : Multiple positive solutions for the boundary value problem of a nonlinear fractional differential equation. Nonlinear Anal. 71, 4676-4688 (2009)

16. Wang, J, Zhou, Y, Wei, W, Xu, H: Nonlocal problems for fractional integrodifferential equations via fractional operators and optimal controls. Comput. Math. Appl. 62, 1427-1441 (2011)

17. Liu, X, Lin, L, Fang, H: Existence of positive solutions for nonlocal boundary value problem of fractional differential equation. Cent. Eur. J. Phys. 11, 1423-1432 (2013)

18. Zhi, E, Liu, X, Li, F: Nonlocal boundary value problem for fractional differential equations with p-Laplacian. Math. Methods Appl. Sci. 37, 2651-2662 (2014)

19. Ntouyas, SK, Etemad, S: On the existence of solutions for fractional differential inclusions with sum and integral boundary conditions. Appl. Math. Comput. 266, 235-243 (2015)

20. Liu, X, Jia, M, Xiang, X: On the solvability of a fractional differential equation model involving the $p$-Laplacian operator. Comput. Math. Appl. 64, 3267-3275 (2012)

21. Jia, M, Liu, X: The existence of positive solutions for fractional differential equations with integral and disturbance parameter in boundary conditions. Abstr. Appl. Anal. 2014, Article ID 131548 (2014)

22. Jia, M, Liu, X: Multiplicity of solutions for integral boundary value problems of fractional differential equations with upper and lower solutions. Appl. Math. Comput. 232, 313-323 (2014)

23. Jia, M, Wang, P: Multiple positive solutions for integro-differential equations with integral boundary conditions and sign changing nonlinearities. Electron. J. Differ. Equ. 2012, 31 (2012)

24. Guo, D, Lakshmikantham, V: Nonlinear Problems in Abstract Cones. Academic Press, New York (1988) 